\title{
Erratum to: Pharmacokinetic Evaluations of the Co-Administrations of Vandetanib and Metformin, Digoxin, Midazolam, Omeprazole or Ranitidine
}

\author{
Susanne Johansson - Jessica Read - Stuart Oliver • \\ Mark Steinberg • Yan Li • Eleanor Lisbon · \\ David Mathews $\cdot$ Philip T. Leese $\cdot$ Paul Martin
}

Published online: 26 September 2014

(c) Springer International Publishing Switzerland 2014

Erratum to: Clin Pharmacokinet (2014) 53:837-847

DOI 10.1007/s40262-014-0161-2

Page 837; column 2, para 4: The first key point, which previously read:

Vandetanib may lead to greater exposure to octamer transcription factor-2 substrates or P-glycoprotein substrates when co-administered.

Should read:

Vandetanib may lead to greater exposure to organic cation transporter 2 substrates or P-glycoprotein substrates when co-administered.

The online version of the original article can be found under doi:10.1007/s40262-014-0161-2.

S. Johansson $(\bowtie)$

AstraZeneca R\&D Mölndal, Quantitative Clinical

Pharmacology, SE-431 83 Mölndal, Sweden

e-mail: susanne.a.johansson@astrazeneca.com

J. Read

PRA International, Reading, UK

S. Oliver · P. Martin

AstraZeneca, Alderley Park, Macclesfield, UK

M. Steinberg $\cdot$ Y. Li

AstraZeneca, Wilmington, DE, USA

E. Lisbon - D. Mathews - P. T. Leese

Quintiles Phase I Services, Overland Park, KS, USA 